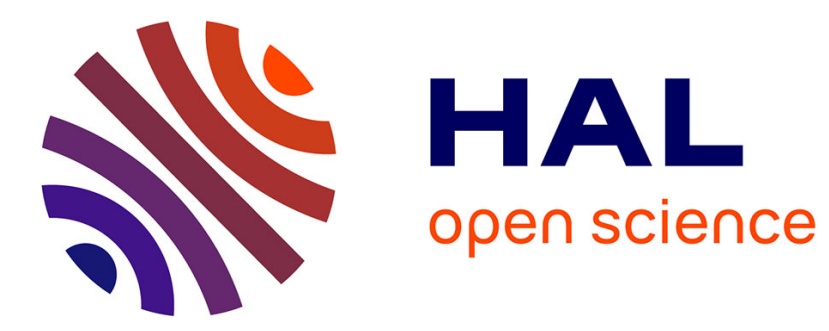

\title{
Influence of relative humidity and loading frequency on the PA6.6 cyclic thermomechanical behavior: Part I. mechanical and thermal aspects
}

\author{
Adil Benaarbia, André Chrysochoos, Gilles Robert
}

\section{To cite this version:}

Adil Benaarbia, André Chrysochoos, Gilles Robert. Influence of relative humidity and loading frequency on the PA6.6 cyclic thermomechanical behavior: Part I. mechanical and thermal aspects. Polymer Testing, 2014, 40, pp.290-298. 10.1016/j.polymertesting.2014.09.019 . hal-01077283

\section{HAL Id: hal-01077283 \\ https://hal.science/hal-01077283}

Submitted on 4 Nov 2014

HAL is a multi-disciplinary open access archive for the deposit and dissemination of scientific research documents, whether they are published or not. The documents may come from teaching and research institutions in France or abroad, or from public or private research centers.
L'archive ouverte pluridisciplinaire HAL, est destinée au dépôt et à la diffusion de documents scientifiques de niveau recherche, publiés ou non, émanant des établissements d'enseignement et de recherche français ou étrangers, des laboratoires publics ou privés. 


\title{
Influence of relative humidity and loading frequency on the PA6.6 cyclic thermomechanical behavior: Part I. mechanical and thermal aspects
}

\author{
Adil Benaarbia a, b, *, André Chrysochoos ${ }^{a}$, Gilles Robert ${ }^{\mathrm{b}}$ \\ a University Montpellier 2, LMGC laboratory, CNRS, Place Eugène Bataillon, 34095 Montpellier, France \\ b Solvay Engineering Plastics, Technyl Innovation Center-Simulation et Validation des Applications, Avenue Ramboz - BP64, 69192 Saint \\ Fons, France
}

\section{Keywords:}

PA6.6 matrix

Relative humidity

Thermoelastic inversion

Self-heating

Quantitative infrared calorimetry

\begin{abstract}
A B S T R A C T
Using quantitative infrared techniques, this study investigated the influence of relative humidity (RH) and loading rate on the mechanical and thermal responses of PA6.6 matrix specimens. The specimens were subjected to tensile and oligocyclic tensile-tensile tests at 3 different RHs. For tensile experiments, the studied samples were loaded at 4 displacement rates. For oligocyclic tensile-tensile fatigue tests, they were subjected to 2 distinct loading frequencies with a 0.1 stress ratio. The overall aim of this study was to analyze the mechanical and thermal responses for each cyclic loading case. The influence of the loading frequency on the hysteresis loops and self-heating was particularly highlighted. The plasticizing role of water and its influence on the glass transition temperature were also discussed relative to the nature of temperature variations accompanying the deformation processes. These thermal responses were associated with dissipation, standard and/or entropic thermoelastic coupling sources. These different heat sources and the complete energy rate balances will be analyzed in the second part of this paper.
\end{abstract}

\section{Introduction}

Thermoplastic polymers are a class of engineering materials of growing commercial importance due to their ease of manufacture and good thermomechanical properties. They have already been successfully used in many applications, especially in the automotive industry. Thermoplastic polymeric materials generally exhibit various deformation mechanisms that occur during monotonous and cyclic loadings, strongly influenced by hygro-thermomechanical boundary and loading conditions (e.g. time effect, hygrothermal sensitivity associated with progressive damage). Most of these mechanisms have been thoroughly

\footnotetext{
* Corresponding author.

E-mail address: abenaarb@univ-montp2.fr (A. Benaarbia).
}

reviewed in several experimental, analytical and numerical investigations (see e.g. [1] for glassy polymers (PMMA) [2], for glass-fiber-reinforced thermoplastic composites based on PA6.6 [3], for thermoplastic polyurethanes (TPUs) [4], for PA6 pure and reinforced with different amounts of short fiber glass [5], for an amorphous polymer (poly(ethylene terephthalate)-glycol (PETG)) [6], for several homopolymers (PA6, PA6.6 and PA46) and [7] for short-glass fiberreinforced PA6.6. These materials are becoming reasonably well known. However, thermomechanical effects tightly connected with these mechanisms have seldom been analyzed, and thus warrant investigation. Further insight is required on some issues, especially concerning the existence of dissipation sources (reflecting the irreversibility of deformation processes) and the competition between standard and entropic thermoelastic couplings, possibly influenced by the loading frequency and relative humidity. 
One promising approach for addressing these issues is based on using quantitative infrared calorimetry techniques. For several years, infrared thermal images have been used to an increasing extent to the thermomechanical behavior of materials $[8,9]$.

This paper focuses specifically on the influence of relative humidity, inducing the so-called water plasticizing effect, on the thermomechanical responses of the polyamide matrix. It is essential to control water plasticizing effects when using polyamide materials for specific industrial applications where the environmental humidity changes considerably. Thermomechanical characterization of these effects could provide crucial information on material changes and, consequently, contribute to modelling of the material's hygro-thermo-mechanical behavior. Unlike metallic materials, some polymers such as nylons, and especially PA6.6, are highly moisture sensitive, thus inducing significant changes in the physicochemical properties of these materials. Indeed, internal moisture may cause these engineering materials to swell (see. e.g. [6]), the amount of swelling being highly dependent on the moisture uptake which, in turn, will be governed by many factors. From a chemical standpoint, water molecules penetrate into the PA6.6 microstructure, thus weakening the intermolecular forces such that the molecular segments become more flexible, with a concomitant decrease in the glass transition temperature [10].

Indeed, water plasticizing effects have been recognized for their marked influence on the glass transition temperature of PA6.6 [11]. Glass transition is a characteristic mechanism which is still under discussion [12-14]. It is a gradual thermally activated process that leads a polymer to change from glassy to rubbery state $[15,16]$. The glass transition temperature, which largely depends on the hygro-thermo-mechanical state, is explained according to many theories as being related to the onset of movements in the macromolecular network of polymers [17]. The addition of water molecules to the polymer microstructure indeed causes a decrease in the glass transition temperature as has, for instance, recently been reported for PA6.6 matrix composites in [18]. Glass transition significantly diminishes as the relative humidity increases, such that PA6.6 shows rubber effects under normal usage temperatures.

This study is fully in line with previous studies on dry PA6.6 specimens. The goal is now to characterize the mechanical, thermal and energy responses of the PA6.6 matrix under different hygro-mechanical loading conditions. Experimental findings were split into two parts: the first dealt with the mechanical and thermal aspects, whereas the second focused on the energy rate balance features. The first part starts with a brief description of the experimental protocol; we discuss the behavior of PA6.6 specimens under different mechanical loadings. We first focus on the mechanical response of the PA6.6 matrix in tensile mode for the 3 studied relative humidity levels and 4 different displacement rates. This is followed by a discussion on the water plasticizing effects on the material behavior. Regarding the cyclic response, the mechanical hysteresis loop forms are analyzed in terms of hysteresis area, mean slope and mean strain per cycle. Thermal responses associated with 2 different loading frequencies are also discussed in terms of dissipative and thermoelastic coupling effects. The contribution of an additional thermomechanical coupling source is also suggested and is basically attributed to entropic elasticity. This first part ends with a discussion on many different mechanical and thermal findings noted during this exploratory work, and heralds the energy aspects developed in the second part.

\section{Experimental set-up and procedures}

\subsection{Material and loadings}

The polymer investigated in this study was a polyamide 6.6 matrix manufactured by Solvay Engineering Polyamides and commercially available under the trade name A218. Test sample dimensions were $100 \times 20 \mathrm{~mm}$ and $3.14 \mathrm{~mm}$ thick (see Fig. 1(a)). After manufacturing, the samples were conditioned in a climatic chamber. Three relative humidity levels that have the most influence on the automotive structures that this material is used for were considered: dry-as-molded (considered as RH0), RH50 (i.e. $50 \%$ relative humidity), and RH80. The final moisture uptake was checked with weight measurements for each relative hygrometry targeted: $0.25 \%$ for $\mathrm{RH} 0,2.8 \%$ for $\mathrm{RH} 50$ and $5.4 \%$ for RH80. All specimens were sealed prior to testing to maintain a controlled uniform humidity.

Tensile and cyclic tensile-tensile tests were carried out using a hydro-controlled MTS testing machine equipped with a $\pm 25 \mathrm{kN}$ load cell. All experiments were conducted at room temperature $\left(23{ }^{\circ} \mathrm{C}\right)$. The tensile tests were performed at different displacement rates ranging from 0.01 to $10 \mathrm{~mm} / \mathrm{s}$. The cyclic tensile-tensile loads were carried out under a stress ratio $R_{\sigma}$ of 0.1 , where $R_{\sigma}=\sigma_{\min } / \sigma_{\max }$. The loading frequencies were $1 \mathrm{~Hz}$ and $10 \mathrm{~Hz}$, while the number of cycles was set at $10^{4}$. The studied cyclic mechanical loading is illustrated in Fig. 1 (b).

\subsection{Temperature field measurement procedure}

As already mentioned, thermographic measurement techniques using an infrared camera are now widespread. The infrared thermal imaging system used in this study was an infrared focal plane array (IRFPA) camera (CEDIP Titanium), cooled by a Stirling system. The camera was used with a $25 \mathrm{~mm}$ lens and contained an InSb detector which is sensitive to infrared 3-5 $\mu \mathrm{m}$ radiation. The storage capacity of the camera was high enough to record successive images throughout the LCF test. It was decided that the detector should be operated with a window size of $160 \times 128$ pixels, a pixel size of $29 \mu \mathrm{m}$ and an integration time of $1800 \mu$ s. The camera frame rate was set at tenfold the loading frequency to get enough sampled data per cycle in order to improve the signal-to-noise ratio using a local least-squares approximation.

During infrared image acquisitions, the temperature measurements can be disturbed by the environmental conditions and the camera orientation. Several cautionary measures were taken in order to limit and mitigate these error sources. The camera, testing machine and computers were turned on at least $4 \mathrm{~h}$ before testing in order to reach 
a

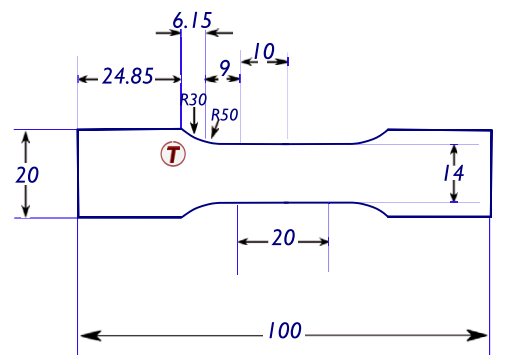

b

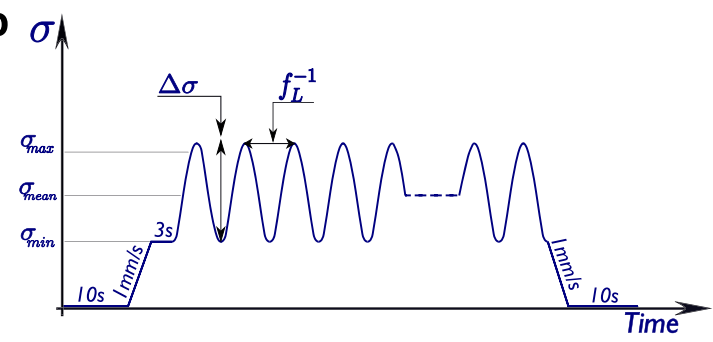

Fig. 1. (a) Shape of the specimens used for the experiments (all dimensions are in millimeters). (b) Diagram of the mechanical loading used in the experiments.

thermal equilibrium. The optical axis of the camera was as perpendicular as possible to the sample surface. Before starting loading, the studied samples were painted with matt black paint to improve and homogenize the surface emissivity; then its value was set at 0.98 , i.e. close to that of a perfect black body. Once the sample was mounted in the gripping system, thermal equilibrium between the sample and its surroundings was reached before starting the test. It is also recommended to operate in the dark or using cold light to limit parasitic IR reflections. The samples were set at $0.5 \mathrm{~m}$ from the camera on a uniform background at constant temperature $\left(\mathrm{T}=23^{\circ} \mathrm{C}\right)$. Unavoidable temperature fluctuations and drifts of the surroundings were tracked using a dummy specimen. Finally, every part of the gripping system close to the sample was screened (i.e. wrapped with paper) and isolated from external heat sources in order to avoid reflections onto the loaded sample.

Parasitic infrared reflections and thermal drifts of the camera and that of the test room were unfortunately not the sole metrological problems facing the IR camera user. Matrix elements of the IR camera detector are not homogeneous. An appropriate pixel calibration protocol was thus conducted to determine the value of the temperature given by each pixel in front of a uniform thermal scene imposed by a black body producing a homogeneous planar energy flux. This allowed conversion of the thermal radiation digitized by the infrared camera into temperature using pixel calibration functions [19].

During the test, several data were recorded through different acquisition set-ups (camera, testing machine, etc.) using different acquisition frequencies ( 10 or $100 \mathrm{~Hz}$ for the IR camera and $102 \mathrm{~Hz}$ for the MTS testing machine). As the frequency acquisitions were chosen to be different, temporal interpolations between both time bases of the infrared and usual mechanical data were necessary to obtain accurate correspondence. A homemade electronic device was designed to directly control these data acquisitions [20].

\section{Experimental results}

\subsection{Tensile tests}

Some mechanical properties of PA6.6 were derived from monotonous uniaxial tensile tests. The stress-strain data obtained from the experiments are presented in terms of normalized engineering stress and engineering strain. In
Fig. 2, typical mechanical responses of tensile tests are plotted for several displacement rates $(10,1,0.1$ and $0.01 \mathrm{~mm} / \mathrm{s}$ ) and hygrothermal states (RHO, RH50 and RH80). The main results are summarized as:

- The results first showed that the maximum reachable load increased when the displacement rate increased. For dry-as-molded samples, the ultimate normalized engineering stress increased by about $10 \%$ when going from $0.01 \mathrm{~mm} / \mathrm{s}$ to $10 \mathrm{~mm} / \mathrm{s}$. Also, the tensile strength of RH50 and RH80 specimens was notably lower than that of the dry-as-molded PA6.6 matrix, indicating softening induced by the plasticizing effect exerted by water. This behavior could be explained by the fact that the water plasticizing action could enhance local molecular mobility, thus reducing the internal forces responsible for molecular interactions and, in turn, leading to a reduction in the elasticity stiffness and an increase in the viscous effects.

- For RH50 and RH80 specimens, the water action started influencing the mechanical response from the outset of tensile test. Hence, no yield stress effect was detectable.

- In addition, macroscopic structural softening was observed (displacement of necking lips), followed by a

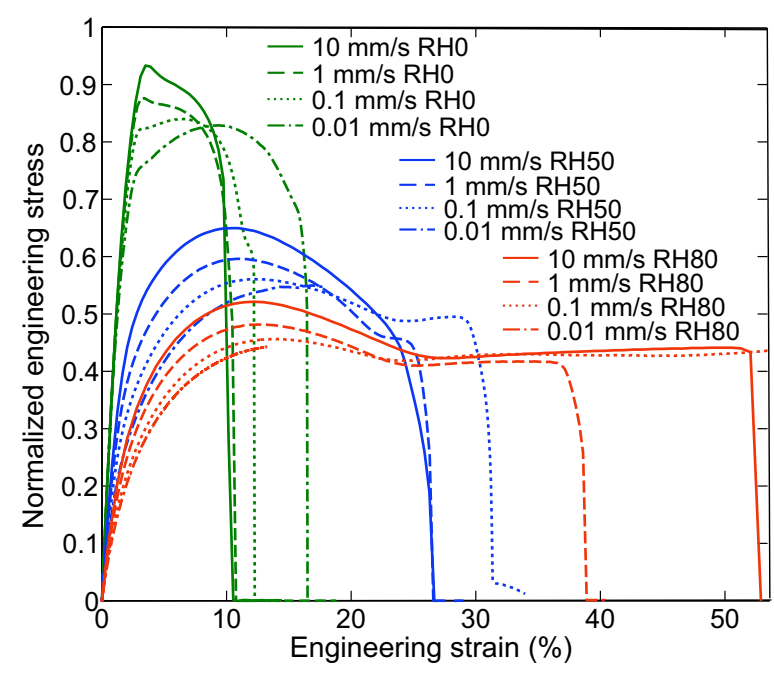

Fig. 2. Normalized engineering stress versus engineering strain curves showing the effect of relative humidity on the tensile properties of PA6.6 matrix subjected to different displacement rates. 
broad plateau, especially for RH80 at all displacement rates $(10 \mathrm{~mm} / \mathrm{s}, 1 \mathrm{~mm} / \mathrm{s}$ and $0.1 \mathrm{~mm} / \mathrm{s})$ and RH50 solely at $0.1 \mathrm{~mm} / \mathrm{s}$. In such cases, because of these structural effects, the stress strain responses plotted in Fig. 2 could no longer be considered as intrinsic material responses. Similar results were recently obtained and widely discussed in [14] for PA6.6 reinforced with $35 \mathrm{wt} \%$ of short glass fibers tested under two hygrometric conditions: dry-as-molded specimens and specimens conditioned at RH50.

- Finally, Fig. 2 shows that the strain level at which the rupture occurred increased considerably with the relative humidity.

\subsection{Cyclic PA6.6 behavior}

\subsubsection{Mechanical response of PA6.6 during oligocyclic fatigue tests}

Cyclic normalized stress-strain curves under non-zero positive mean stress $\left(R_{\sigma}=0.1\right)$ and different water contents (RH0, RH50, RH80) were plotted in Fig. 3. In each case, we observed the hysteretic response. We introduced 3 parameters to characterize variations in the hysteresis loop:

- the hysteresis area, which represents the deformation energy lost within a complete loading cycle,

- the mean slope of the hysteresis loop, which can be interpreted as material stiffness,

- the mean strain over a complete cycle, which reflects the creep induced by nonzero mean stress.

Note that different gains were applied to mechanical data to distinctly plot the time courses of these parameters on the same figure (Fig. 3 (g-h-i)). These gains (denoted by $\mathrm{G})$ are directly indicated on each curve.

- The hysteresis loops were clearly much thinner for dryas-molded specimens (Fig. 3 (a-d)) than that in high water content specimens (Fig. 3 (b-c-e-f)). We also observed that the hysteresis area decreased, and this trend was more marked at high relative humidity and low strain rate (Fig. 3 (g))

- The change in the mean slope was, in our opinion, due to both the effect of relative humidity and/or cyclic fatigue. We first observed that this mean slope markedly decreased at the beginning of tests for all water contents and strain rates (Fig. 3 (h)). In some cases, the slope seemed to stabilize but never before a few hundred cycles.

- Moreover, a ratcheting phenomenon characterized by drift in the mean strain per cycle was observed. The shifting of hysteresis loops, indicated in all of the selected figures, was less pronounced for dry-as-molded specimens (see. Fig. 3 (a-d-i)), whereas it was significant for both RH50 and RH80 specimens (Fig. 3 (b-c-e-f-i)).

- We finally observed that, although changes in the hysteresis loop and in the mean strain per cycle were less pronounced after 5000 cycles, the hysteresis loop area never stabilized completely.
All of these features showed that the cyclic stabilization of the 3 parameters characterizing the specimen cyclic response never occurred and, when it could be reasonably considered that a parameter was quasi-stabilized, this only occurred after a few hundred cycles. All of these observations led us to consider that the anelastic shakedown concept often invoked in fatigue criteria is irrelevant for PA6.6.

\subsubsection{Thermal response of PA6.6 during oligocyclic fatigue tests}

Fig. 4 shows spatial averages over the specimen gauge part of temperature variations as a function of the number of cycles for a PA6.6 matrix conditioned with the 3 different RH subjected to 2 loading frequencies $\left(f_{L}=1 \mathrm{~Hz}\right.$ and $f_{L}=10 \mathrm{~Hz}$ ).

- Fig. 4 (a-b-c) shows that temperature variations increased rapidly at the very beginning of the $1 \mathrm{~Hz}$ fatigue test, reaching a maximum (black dashed circle for all selected plots), with values ranging from $2.5^{\circ} \mathrm{C}, 16^{\circ} \mathrm{C}$ to $30{ }^{\circ} \mathrm{C}$ for RH0, RH50 and RH80 specimens, respectively. This noticeable self-heating should certainly be closer to the rapid changes of the 3 previous parameters (the hysteresis area, the mean slope and strain) characterizing the cyclic evolution of the hysteresis loop.

- Subsequently, the temperature slowly decreased, probably due to a slowdown in the time pattern of the hysteresis curve parameters. Indeed, a temperature decrease could be related to a decrease of the dissipation intensity cumulated with heat exchanges between the specimen and the surroundings.

- Note that in the case of a "high" loading frequency $\left(f_{L}=10 \mathrm{~Hz}\right)$, the thermal plots showed that the temperature decrease was lower than for «low» tests (Fig. 4 (f-g)), and actually did not occur (Fig. 4 (d-e)) for dry specimens.

- The marked influence of the relative humidity on the thermal effects should be emphasised. Note that the selfheating of dry specimens was approximately tenfold lower than for moist ones, for the same stress range and loading frequency. This result is of crucial importance when considering the thermal sensitivity of the PA6.6 behavior. Cyclic tests cannot be considered as isothermal mechanical tests from a modeling standpoint.

- Note finally that temperature variations were induced by two independent types of heat source: dissipation and coupling sources. The intrinsic dissipation was attributed to the material irreversibility and was nonnegative (2nd principle of thermodynamics). Regarding the coupling mechanisms, two coupling effects should be noted: (a) Standard thermoelastic effects, first observed by Lord Kelvin (1857) [21], reflect the thermodilatability of the material and could be detected via symptomatic thermal oscillations out of phase with the monochromatic loading signal. (b) Rubber elasticity, also termed "entropic elasticity", first observed by Gough (1805) [22], was related to the stress-induced orientation/disorientation of macromolecular chains, leading to reversible oscillation of the conformation entropy and then to temperature oscillation in phase with the monochromatic loading signal. 

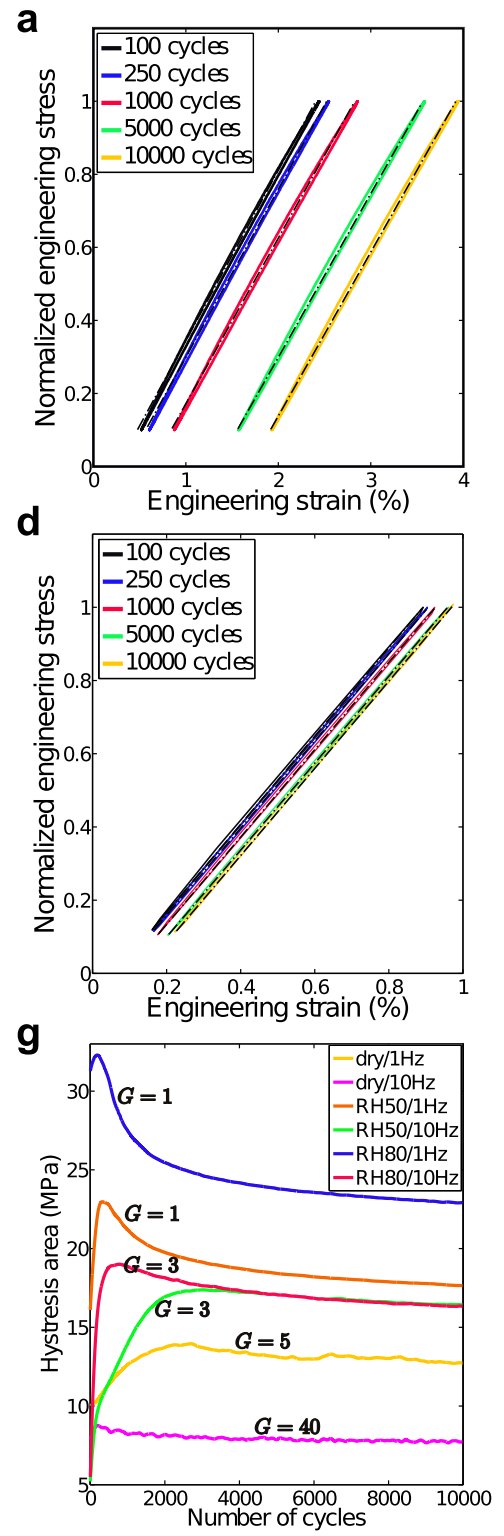
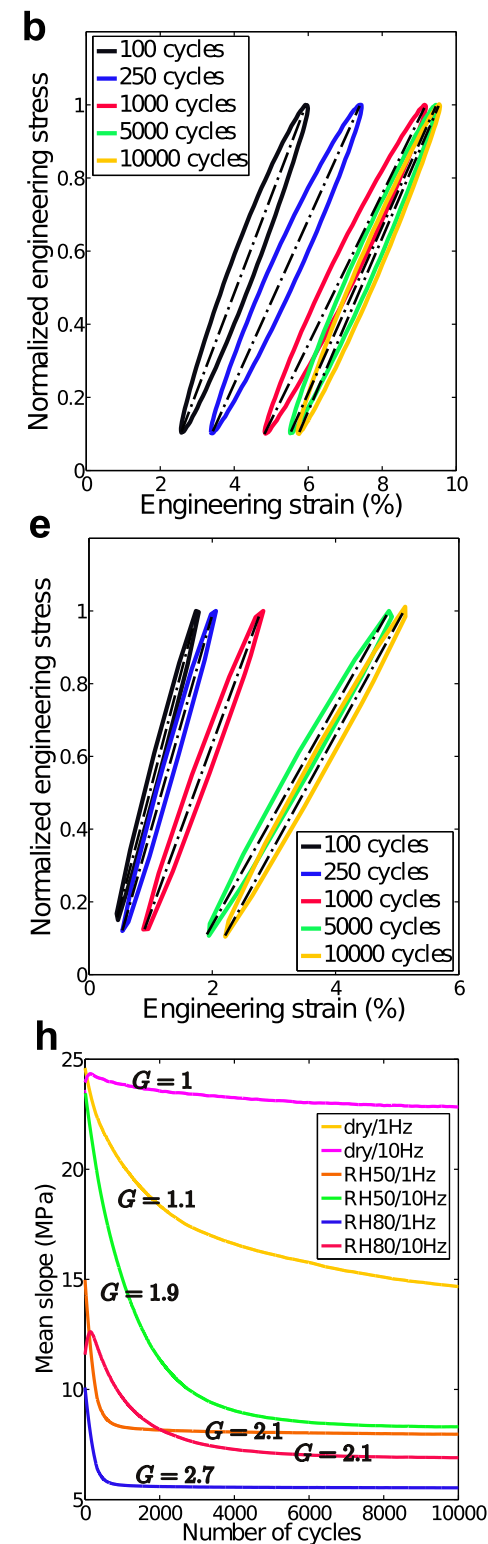
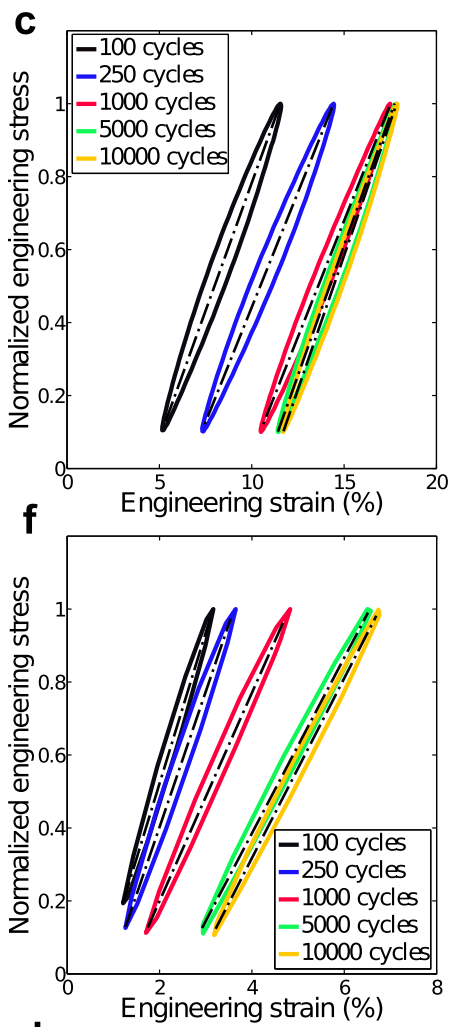

i

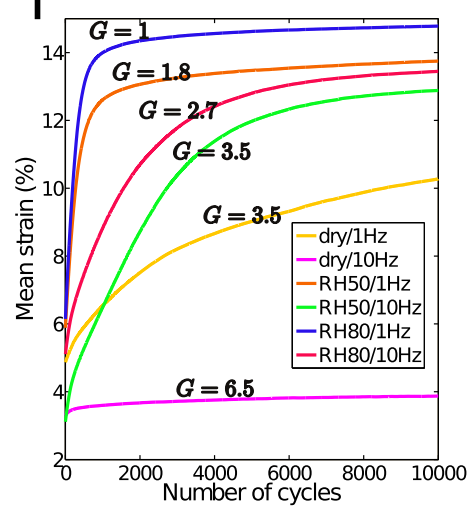

Fig. 3. Normalized engineering stress versus engineering strain curves showing the effect of internal relative humidity on the cyclic mechanical properties of the PA6.6 matrix (a) RHO/1Hz, (b) RH50/1Hz, (c) RH80/1Hz, (d) RHO/10Hz, (e) RH50/10Hz and (f) RH80/10Hz. The plots (g), (h) and (i) represent the hysteresis area, the mean slope and the mean strain per cycle as a function of the number of cycles for all tests. Note that stress normalization was done for each test.

Possible competition between these two thermomechanical couplings is generally associated with the famous "thermoelastic inversion" effect first shown by [23] and later by [24]. Several attempts to model this thermoelastic inversion have been reported. This modeling framework is called "modified entropic elasticity" and belongs to the nonlinear hyperelasticity domain $[25,26]$. Fig. 4 shows the oscillations that occurred within the temperature variation range throughout the cycle. In each case, we specify whether these oscillations were in phase or out of phase with the loading signal.

More precisely, Fig. 5 shows typical evidence of this thermoelastic inversion for a PA6.6 matrix conditioned at RH80 during a tensile-tensile fatigue test at $1 \mathrm{~Hz}$. Both the normalized applied force and normalized temperature variations were plotted in order to show this thermoelastic inversion. At the beginning of cyclic loading, the thermosignal in Fig. 5 (a) increased (until point A) with tensile unloading (until point B) and conversely decreased during loading. In this case, the standard thermoelasticity was predominant. However, beyond a certain number of cycles, and because of ratcheting, which induced gradual elongation of the specimen, the temperature variations started to increase with the tensile loading and then decreased during unloading (see Fig. 5 (b)). This reflects the fact that entropic effects became prevalent. A simple heuristic description of the thermoelastic inversion is proposed hereafter. However, this proposed modeling focuses only 

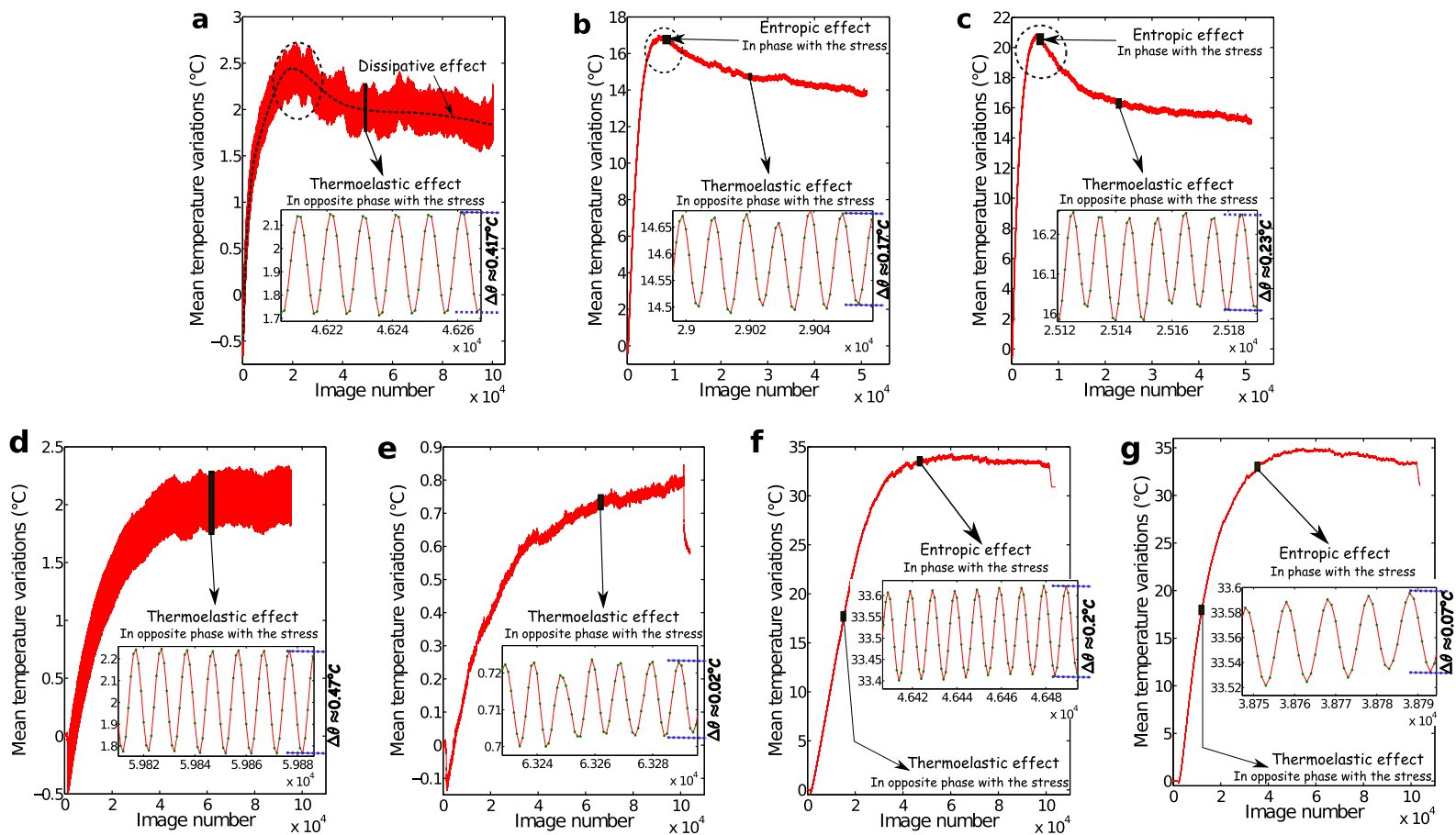

Fig. 4. Spatial averages of temperature variations for PA6.6 matrix specimens: (a) RHO/1Hz, (b) RH50/1Hz, (c) RH80/1Hz, (d) RHO/10Hz/high stress range, (e) RHO/ $10 \mathrm{~Hz} /$ low stress range, (f) $\mathrm{RH} 50 / 10 \mathrm{~Hz}$ and (g) $\mathrm{RH} 80 / 10 \mathrm{~Hz}$. The subplots highlight temperature oscillations during the cycles.

on the coupling effects. Complete thermomechanical modeling should naturally take the fatigue dissipative effects into account. Such work is premature and is beyond the scope of the present paper.

\section{Some comments on entropic vs. standard thermoelasticity}

Several physical approaches have been developed in the past to describe so-called rubber hyper-elasticity. The pioneering studies of Treloar should be mentioned [27,28]. In these works, a model based on statistical molecular theory was developed, where the stress appeared to be proportional to the temperature. Many other constitutive theories were then developed, such as the important works of Flory and Erman (1982) [29], Chadwick and Creasy (1984) [25] and Arruda and Boyce (1993) [30].

In the following, we depict thermoelastic inversion as a simple superposition of standard and entropic thermoelasticity using the Generalized Standard Material formalism (GSM) introduced by [31]. The challenge here is to be in accordance with the statistical formulation of the rubber elasticity, while also being in position to use the GSM approach to formulate the constitutive equations of PA6.6, which are particularly suitable for introducing new constitutive models in computer-aided design tools. Based
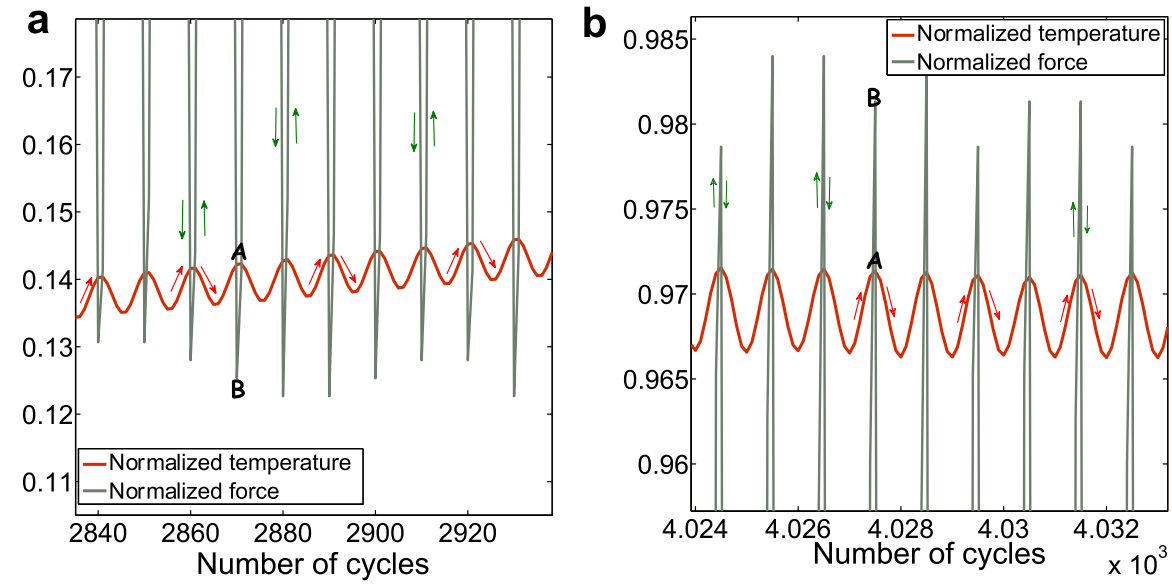

Fig. 5. Normalized force and normalized spatially mean temperature variations as a function of the cycling number, showing the thermoelastic inversion effect; (a) opposite phase: standard thermoelasticity prevalent; (b) in phase: entropic elasticity prevalent. 
on the assumption formulated by [28], the internal energy $e_{r}$ of an entropic elastic material, which is usually a function of entropy $s$ and strain $\boldsymbol{\varepsilon}$, is solely a function of the absolute temperature $\tilde{e}(T)$, i.e. a typical characteristic of an ideal gas:

$e_{r}(s, \boldsymbol{\varepsilon})=\tilde{e}(T)$

We classically define the absolute temperature as $T=e_{r, s}=\partial e_{r} / \partial s$ and the reversible stress tensor $\boldsymbol{\sigma}^{r}=\boldsymbol{e}_{r, \varepsilon}$. Recall that, in the thermoelasticity framework, no intrinsic dissipation occurs, so the irreversible stress $\sigma^{i r}$ vanishes. Consequently, the reversible stress $\sigma^{r}$ can be identified by the stress tensor $\boldsymbol{\sigma}$. The time derivative of the specific internal energy then reads:

$\dot{e}_{r}=T \dot{s}+\boldsymbol{\sigma}: \dot{\boldsymbol{\varepsilon}}=\tilde{e}^{\prime} \dot{T}$

The symbol $\boldsymbol{x}^{\prime}$ denotes the derivative of $\boldsymbol{x}$ with respect to the absolute temperature $T$. When introducing the Helmholtz free energy of rubber materials $\psi_{r}=e_{r}-T s$, the time derivative of the specific entropy $s=-\left(\psi_{r}\right)_{T}$ reads:
This implies that microstructural mechanisms of polymeric chains such as micro-motions, slippages and entanglements cannot be taken into account. To do so, other potentials have to be considered. For instance, the Edwards and Vilgis modeling approach was used by [32] to predict the dynamic behavior of PA6.6 at large strains. The free energy considered in Eq. 5 was derived from the Flory affine model [33], which does not take the chain extensibility limitation into account. However, we estimated that this model could give sufficiently satisfactory results for moderate strain ranges.

The part of the PA6.6 free energy dedicated to thermoelastic couplings can be seen as the sum of the free energy $\psi_{\text {the }}$ defined for a standard thermoelastic material and that of a rubber material $\psi_{r}$. Then, when considering the Hencky (logarithmic) strain, we supposed that the total strain tensor could be split into an elastic part $\varepsilon_{e}$ and rubber part $\boldsymbol{\varepsilon}_{r}$, as is the case for any series model. For the 1D model with incompressible rubber transformation, the overall specific energy could then be written as:

$$
\psi\left(\theta, \varepsilon, \varepsilon_{r}\right)=\frac{1}{2} \underbrace{E\left(\varepsilon-\varepsilon_{r}-\alpha_{t h} \theta\right)^{2}-\overbrace{\frac{1}{2}\left(\frac{\rho C}{T_{r}}+E \alpha_{t h}^{2}\right) \theta^{2}}^{\psi_{r}}+\mathcal{K}_{r}\left(\theta+T_{r}\right)\left(\frac{e^{2 \varepsilon_{r}}}{2}+e^{-\varepsilon_{r}}-\frac{3}{2}\right)}_{\psi_{\text {the }}}
$$

$\dot{s}=-\left(\psi_{r}\right)_{T, T} \dot{T}-\left(\psi_{r}\right)_{,, T}: \dot{\varepsilon}$

When inserting Eq. 3 into Eq. 2, we get:

$\underbrace{\left(\tilde{e}^{\prime}-C_{\varepsilon}\right)}_{\left(E_{2}\right)} \dot{T}-\underbrace{\left(T s_{, \varepsilon}+\boldsymbol{\sigma}\right)}_{\left(E_{1}\right)}: \dot{\boldsymbol{\varepsilon}}=0$

If Eq. 4 equals zero, irrespective of the process (i.e. $\forall(\dot{T}, \dot{\boldsymbol{\varepsilon}})$ ), E1 and E2 must also be equal to zero. The first subequation indicates that the specific heat $C_{\varepsilon}$ is a unique function of the temperature (typical ideal gas). The second equation shows that the stress for a rubber polymer is proportional to the temperature. This relation has the same form as the statistical macromolecular theory [27].

Since $T\left(\psi_{r}\right)_{, \varepsilon, T}=\left(\psi_{r}\right)_{, \varepsilon}$, it can readily be shown that the specific free energy $\psi_{r}$ takes the following form:

$\psi_{r}(T, \boldsymbol{\varepsilon})=T \mathscr{K}_{1}(\boldsymbol{\varepsilon})+\mathscr{K}_{2}(T)$

where $\mathscr{K}_{1}$ and $\mathscr{K}_{2}$ are integration functions. The choice of such entropic free energy satisfies both Eq. 2 and Eq. 4-E2. For simplicity, we chose the Gaussian model in order to determine the function $\mathscr{K}_{1}$. Moreover, the second contribution $\mathscr{K}_{2}$ was determined while assuming that the specific heat $C_{\varepsilon}$ would be constant in the vicinity of the thermal equilibrium. Hence, we took the free energy variations directly connected to temperature variations into account. where $E$ stands for the elastic modulus, $\alpha_{t h}$ the thermal expansion coefficient, $\theta=T-T_{r}$ the change in the current temperature $T$, where $T_{r}$ represents the room temperature and $\mathscr{K}_{r}$ a material constant.

In agreement with the foregoing, the heat sources could thus be derived from Eq. 6. With a simplified OD heat diffusion model, the novel heat equation becomes:

$\rho\left(\langle\dot{\theta}\rangle+\frac{\langle\theta\rangle}{\tau_{\text {th }}^{0 D}}\right)=-\left\langle E \alpha_{t h} T\left(\dot{\varepsilon}-\dot{\varepsilon}_{r}\right)\right\rangle+\left\langle\mathscr{K}_{r} T\left(e^{2 \varepsilon_{r}}-e^{-\varepsilon_{r}}\right) \dot{\varepsilon}_{r}\right\rangle$

Two coupling sources are now involved on the right hand side of the heat diffusion equation. The first source characterizes the mean standard thermoelastic effects while the second deals with the rubber effects.

As long as the temperature variations remain small with respect to the absolute temperature (isothermal approximation), it can be shown that the mean coupling sources averaged over a complete mechanical cycle remain equal to zero:

$$
\lim _{\substack{T(\tau) \rightarrow T_{r} \\ \forall \tau \in\left[t, t+1 / f_{L}\right]}}\left\langle\int_{t}^{t+\frac{1}{f_{L}}} \mathscr{W}_{c}^{\prime} d \tau\right\rangle=0
$$

where $f_{L}$ is the loading frequency. 
In practice, for PA6.6, this mean energy induced by couplings remained low compared to the size of the hysteresis loop. This naturally does not mean that the corresponding energy rates were negligible. On the contrary, the range of the thermoelastic coupling sources may become even more than several hundredfold greater than the mean dissipation intensities per cycle. This is commonly the case for metallic materials [34]. For some other materials (e.g. shape memory alloys), it has been shown that such coupling mechanisms could be the main cause of triggering a hysteretic mechanical response [35].

\section{Discussion}

In this first part, we analyzed the influence of relative humidity and loading rate, focusing on the mechanical and thermal responses of the semi-crystalline PA6.6. The findings showed that the relative humidity conditions and loading frequency completely affected the shape and drift of the mechanical hysteresis loops. The loops were much thinner for dry-as-molded specimens (resp. low loading frequency) than for high water content specimens (resp. high loading frequency). The drift in hysteresis loops was less pronounced for dry-as-molded specimens (resp. high loading frequency), whereas it was significant for both humid specimens (resp. low loading frequency). Regarding the thermal responses, it was shown that the self-heating levels of dry specimens were approximately tenfold that for humid ones. We also noted that temperature variations were induced by two independent types of heat source: dissipation and coupling sources. At this stage, two coupling effects were also characterized: standard thermoelastic effects (for low relative humidity rates and/or small elongations) and rubber elasticity (for high relative humidity rates and large elongations). Finally, a simple heuristic description of the competition (e.g. thermoelastic inversion) of these two coupling sources was proposed.

The second part of this work will use these mechanical and thermal data to draw up complete energy rate balances associated with the experiments. Such energy balances can provide better insight into the evolving of the physical phenomena that cause the temperature increase (or decrease) observed when PA6.6 matrix is subjected to cyclic loadings. These energy analyses should also assess the role of humidity and loading frequency on the thermodynamic signatures of the deformation processes accompanying cyclic loading of the PA6.6 matrix.

\section{Acknowledgements}

The authors gratefully acknowledge Solvay Engineering Plastics for supporting this work and for providing material data and specimens. This work benefited from the financial support of the French Minister for Research (ANRT), CNRS 079212:UM2 121532 and was performed in the framework of the European DURAFIP project (FUI project supported by Oseo).

The authors also wish to warmly thank a very present but discrete partner, David Manley, for the many effective tips that significantly improved the presentation of our work.

\section{References}

[1] E.M. Arruda, M.C. Boyce, R. Jayachandran, Effects of strain rate, temperature, and thermo-mechanical coupling on the large strain deformation of glassy polymers, Mechanics of Materials 19 (1995) $193-212$.

[2] A. Bergeret, I. Pires, M.P. Foulc, B. Abadie, L. Ferry, A. Crespy, The hygrothermal behaviour of glass-fibre reinforced thermoplastic composites: a prediction of the composite lifetime, Polymer Testing 20 (2001) 753-763.

[3] J. Qi, M.C. Boyce, Stress-strain behavior of thermoplastic polyurethane, Mechanics of Materials 31 (2005) 817-839.

[4] I. Carrascal, J.A. Casado, J.A. Polanco, F. Gutierrez-Solana, Absorption and diffusion of humidity in fiber glass-reinforced polyamide, Polymer Composites 26 (2005) 580-586.

[5] R.B. Dupaix, M.C. Boyce, Constitutive modeling of the finite strain behavior of amorphous polymers in and above the glass transition, Mechanics of Materials 39 (2007) 39-52.

[6] L. Monson, M. Braunwarth, C.W. Extrand, Moisture absorption by various polyamides and their associated dimensional changes, Journal of Applied Polymer Science 107 (2008) 355-363.

[7] M.F. Arif, F. Meraghni, Y. Chemisky, N. Despringre, G. Robert, In situ damage mechanisms investigation of PA66/GF30 composite: effect of relative humidity, Composites Part B: Engineering 58 (2014) 487-495.

[8] A. Chrysochoos, H. Louche, An infrared image processing to analyse the calorific effects accompanying strain localization, International Journal of Engineering Science 38 (2000) 1759-1788.

[9] B. Berthel, A. Chrysochoos, W. Wattrisse, A. Galtier, Infrared image processing for the calorimetric analysis of fatigue phenomena, Experimental Mechanics 48 (2008) 79-90.

[10] G. Erhard, Designing with Plastics, Hanser Gardner Publications, 2006.

[11] J. Brandrup, E.H. Immergut, E.A. Grulke, Polymer Handbook, fourth ed., J. Wiley \& Sons., Inc, New York, 1999.

[12] A. Hassan, N.M. Salleh, R. Yahya, M.R.K. Sheikh, Fiber length, thermal, mechanical, and dynamic mechanical properties of injectionmolded glass-fiber/polyamide 6.6: plasticization effect, Journal of Reinforced Plastics and Composites 30 (2011) 488-498.

[13] A. Hassan, N.A. Rahman, R. Yahya, Moisture absorption effect on thermal, dynamic mechanical and mechanical properties of injection-molded short glass-fiber/polyamide 6.6 composites, Fibers and Polymers 13 (2012) 899-906.

[14] A. Launay, M.H. Maitournam, Y. Marco, I. Raoult, F. Szmytka, Cyclic behaviour of short glass fibre reinforced polyamide: experimental study and constitutive equations, International Journal of Plasticity 27 (2011) 1267-1293.

[15] L.H. Sperling, Introduction to Physical Polymer Science, second ed., J. Wiley \& Sons., Inc., New York, 1992.

[16] F. Rodriguez, Principles of Polymer Systems, fourth ed., Taylor \& Francis, Washington, DC, 1996. Chapter 13 (Recycling and Resource Recovery).

[17] V.N. Bliznyuk, F.E. Assender, G.A.D. Briggs, Surface glass transition temperature of amorphous polymers. A new insight with SFM, Macromolecules 35 (2002) 6613-6622.

[18] A. Launay, Y. Marco, M.H. Maitournam, I. Raoult, Modelling the influence of temperature and relative humidity on the timedependent mechanical behaviour of a short glass fibre reinforced polyamide, Mechanics of Materials 56 (2013) 1-10.

[19] V. Honorat, S. Moreau, J.M. Muracciole, B. Wattrisse, A. Chrysochoos, Calorimetric analysis of polymer behaviour using a pixel calibration of an IRFPA camera, Qirt Journal 2 (2005) 153-171.

[20] A. Chrysochoos, B. Wattrisse, J.M. Muracciole, Y. El Kaim, Fields of stored energy associated with localized necking of steel, Journal of Mechanics of Materials and Structures 4 (2009) 245-262.

[21] W. Thomson, Lord Kelvin, On the thermo-elastic and thermomagnetic properties of matter, Part I (dated 10th March, 1855), Quarterly Journal of Mathematics (1857) 57-77.

[22] W. Gough, 2nd ser., Manchester Literary and Philosophical Society, 1,1805, p. 288

[23] W. Joule, 4th Ser., On Some Thermodynamic Properties of Solids, Philosophical Magazine, 14, Taylor \& Francis, 1857, p. 227

[24] R.L. Anthony, R.H. Caston, E. Guth, Equations of state for naturals and synthetic rubber like materials: unaccelerated natural soft rubber, Journal of Physical Chemistry 46 (1942) 826.

[25] P. Chadwick, C.D.M. Creasy, Modified entropic elasticity of rubberlike materials, Journal of Mechanics of Physics and Solids 32 (1984) 337-397.

[26] R.W. Ogden, Aspects of the phenomenological theory of rubber thermoelasticity, Polymer 28 (1987) 379. 
[27] L.R.G. Treloar, The elasticity and related properties of rubbers, Reports on Progress in Physics 36 (1973) 755-826.

[28] L.R.G. Treloar, The Physics of Rubber Elasticity, third ed., Clarendon press, Oxford, 1975.

[29] P.J. Flory, B. Erman, Theory of elasticity of polymer networks, Macromolecules 15 (1982) 800-806.

[30] E.M. Arruda, M.C. Boyce, A three-dimensional model for the large stretch behavior of rubber elastic materials, Journal of Mechanics and Physics of Solids 41 (1993) 389-412.

[31] B. Halphen, Q.S. Nguyen, On the generalized standards materials (in French), Journal de Mécanique 14 (1975) 39-63.

[32] N. Billon, Newconstitutive modeling for time-dependent mechanical behavior of polymers close to glass transition: fundamentals and experimental validation, Journal of Applied Polymer Science 125 (2012) 4390-4401.

[33] P.J. Flory, J. Rehner, Statistical mechanics of crosslinked polyme networks I. Rubberlike elasticity, Journal of Chemical Physics 11 (1943) 512-520.

[34] B. Berthel, B. Wattrisse, A. Chrysochoos, A. Galtier, Thermographic analysis of fatigue dissipation properties of steel sheet, Strain 43 (2007) 273-279.

[35] A. Chrysochoos, Thermomechanical analysis of the cyclic behavior of materials, Procedia IUTAM 4 (2012) 15-26. 